\title{
Influence of Pressure on the Magnetic Response of the Low-Dimensional Quantum Magnet $\mathrm{Cu}\left(\mathrm{H}_{2} \mathrm{O}\right)_{2}\left(\mathrm{C}_{2} \mathrm{H}_{8} \mathrm{~N}_{2}\right) \mathrm{SO}_{4}$
}

\author{
M.K. Peprah ${ }^{a}$, D. VanGennep ${ }^{a}$, B.D. Blasiola ${ }^{a}$, P.A. Quintero ${ }^{a}$, R. Tarasenko ${ }^{b}$, \\ J.S. XiA ${ }^{a}$, J.J. HAMlin ${ }^{a}$, M.W. MeISEL ${ }^{a, b, *}$ AND A. OREndÁČOVÁ ${ }^{b}$ \\ ${ }^{a}$ Department of Physics and National High Magnetic Field Laboratory, University of Florida, \\ Gainesville, FL 32611-8440, USA \\ ${ }^{b}$ Institute of Physics, Faculty of Science, P.J. Šafárik University, Park Angelinum 9, 04154 Košice, Slovakia \\ The influence of pressure on the low-dimensional molecular magnet $\mathrm{Cu}\left(\mathrm{H}_{2} \mathrm{O}\right)_{2}(\mathrm{en}) \mathrm{SO}_{4}$ (en = ethylenedia- \\ mine $=\mathrm{C}_{2} \mathrm{H}_{8} \mathrm{~N}_{2}$ ) has theoretically been shown to affect the exchange interactions of the material. Herein, the \\ results of an experimental study of hydrostatic pressure effects on the temperature dependence of the magnetiza- \\ tion are reported. Using two different pressure cells, the magnetization measurements were performed between $2 \mathrm{~K}$ \\ and $9.6 \mathrm{~K}$ with pressures ranging from ambient to $5.0 \mathrm{GPa}$. The data preliminarily suggest the presence of a shift \\ in the magnetization peak of the material at the lowest temperatures and at the highest applied pressures. These \\ data serve as a guide for future experimental work employing pressure to study this intriguing system.
}

DOI: 10.12693/APhysPolA.131.901

PACS/topics: 75.40.Cx, 75.50.Ee, 74.62.Fj, 07.35.+k

\section{Introduction}

One approach to new materials discovery involves enhanced interactive collaborations between experimental and theoretical research teams. For example, experimental studies identified $\mathrm{Cu}\left(\mathrm{H}_{2} \mathrm{O}\right)_{2}($ en $) \mathrm{SO}_{4}$ (en = ethylenediamine $=\mathrm{C}_{2} \mathrm{H}_{8} \mathrm{~N}_{2}$ ) as a quasi-two-dimensional $S=1 / 2$ spatially-anisotropic triangular-lattice antiferromagnet, Fig. $1[1,2]$. On the other hand, a theoretical $a b$ initio investigation of the exchange interactions between $\mathrm{Cu}$ ions indicated the system is a quasi-one dimensional magnet $[3,4]$, with the exchange coupling between the $\mathrm{Cu}$ atoms being propagated along a zig-zag line in the $b-c$ plane and connecting the $\mathrm{Cu}$ atoms through the $\mathrm{N}$ atoms, Fig. 1. These numerical studies were extended to pressures up to $8.2 \mathrm{GPa}$, thereby allowing the pressure dependence of the calculated exchange interactions to be predicted [4]. Indeed, at the highest pressures, the predicted one-dimensional nature of the magnetic interactions evolves to possess a two-dimensional character. The purpose of the present investigation was to study the magnetic response of a single crystal sample of $\mathrm{Cu}\left(\mathrm{H}_{2} \mathrm{O}\right)_{2}(\mathrm{en}) \mathrm{SO}_{4}$, hereafter referred to as CUEN, to high pressures and to compare the results with the theoretical predictions. Using two different pressure cells, magnetization measurements were performed between $2 \mathrm{~K}$ and $9.6 \mathrm{~K}$ with pressures ranging from ambient to $5.0 \mathrm{GPa}$. The data suggest a possible a shift in the magnetization peak of the material at the lowest temperatures and at the highest applied pressures.

*corresponding author; e-mail: meisel@phys.ufl.edu

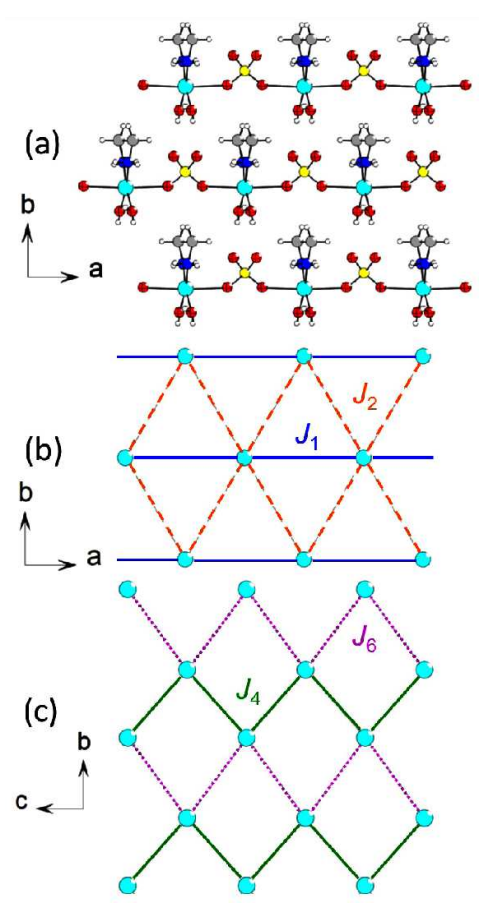

Fig. 1. $\mathrm{Cu}\left(\mathrm{H}_{2} \mathrm{O}\right)_{2}$ (en) $\mathrm{SO}_{4}$ (CUEN). (a) The crystal structure ( $a-b$ plane shown) has a monoclinic, triangular lattice with unit cell parameters $a=7.232 \AA$, $b=11.725 \AA, c=9.768 \AA, \beta=105.50^{\circ}$, and $Z=4$ [1]. The elements are $\mathrm{Cu}$ (light blue), $\mathrm{O}$ (red), $\mathrm{N}$ (dark blue), C (gray), S (yellow), and $\mathrm{H}$ (white). (b) The previous experimental studies interpreted the magnetic response in terms of a two-dimensional triangularlattice with interaction parameters $J_{1}$ and $J_{2}$ in the $a-b$ plane $[1,2]$. (c) The theoretical ab initio work used six, magnetic exchange parameters and found $J_{4}$, in the $b-c$ plane, to be the largest by a factor of about 7 compared to the next largest exchange, $J_{6}$, at $P=0$, resulting in an one-dimensional zig-zag chain system $[3,4]$. At $P=8.2 \mathrm{GPa}$, the ratio $J_{4} / J_{6}=3$, so a two-dimensional magnetic system is predicted [4]. 


\section{Experimental techniques}

The synthesis and structural characterizations of the large crystals $\mathrm{Cu}\left(\mathrm{H}_{2} \mathrm{O}\right)_{2}\left(\mathrm{C}_{2} \mathrm{H}_{8} \mathrm{~N}_{2}\right) \mathrm{SO}_{4}$ are described elsewhere [2]. For each run, small pieces or flakes from the large parent crystals were used.

All of the data discussed in this paper were collected using a commercial magnetometer (Quantum Design MPMS-XL7) capable of generating magnetic fields up to $7 \mathrm{~T}$ and operating down to $2 \mathrm{~K}$. Since the present study was an initial investigation, data were collected at temperatures, $T$, in the range $2 \mathrm{~K} \leq T \leq 9.6 \mathrm{~K}$, and the magnetic field was fixed at $1 \mathrm{kG}$. Depending on the experimental run and pressure cell being employed, the background contributions were subtracted from the raw signal by using the automatic background subtraction (ABS) algorithm when using the piston pressure cell or making a point-by-point subtraction when using the anvil pressure cell only. In both instances, the pressure cell sans sample was measured to provide a typical background signal.

The piston pressure cell, primarily constructed from $\mathrm{BeCu}$, was custom-designed and constructed at the University of Florida [5] and used the superconducting transition temperature of $\mathrm{Pb}$ as a manometer $[6,7]$ and Daphne-7373 oil as a pressure transmission medium [812]. The maximum pressure for this cell is approximately 1.4 GPa, because at higher pressures, the parts of the cell irreversibly deform. The mass of the sample used for this cell was $4 \mathrm{mg}$, and the pressure study was performed for $0 \leq P \leq 0.6 \mathrm{GPa}$.

The anvil pressure cell, primarily constructed from $\mathrm{CuTi}$, was constructed at the University of Florida [5] and was based on a Tozer turnbuckle design [13, 14]. Although diamonds can be utilized, the anvils for this work were $\mathrm{SiC}$ with $800 \mu \mathrm{m}$ diameter culets. $\mathrm{A} \mathrm{BeCu}$ gasket, with a hole diameter of $290 \mu \mathrm{m}$ confined the sample, Ruby $\left(\mathrm{Al}_{2} \mathrm{O}_{3}\right)$ fluorescence served as a manometer $[15,16]$, and Daphne-7373 was employed as the pressure transmitting fluid [8-12]. The fluorescence spectra,

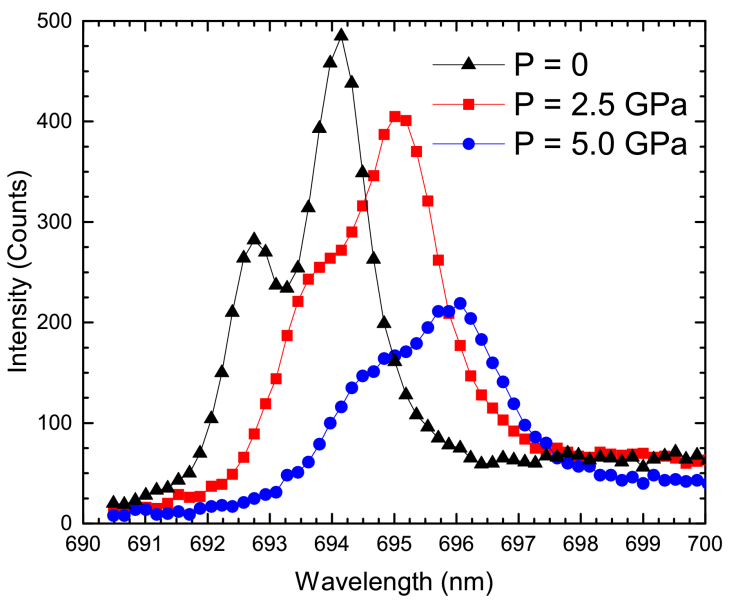

Fig. 2. The wavelength dependence of the intensity of the fluorescence of the ruby in the anvil pressure cell at room temperature.
Fig. 2, were acquired by a home-made, inexpensive minispectrometer [17]. The mass of the sample used for this cell was $\approx 4 \mu \mathrm{g}$, and the pressure was varied in the range $0 \leq P \leq 5.0 \mathrm{GPa}$.

\section{Results and discussion}

The results of this experimental study of the pressure and temperature dependences of the low-field $(1 \mathrm{kG})$ magnetization, $M(P \leq 5 \mathrm{GPa}, 2 \mathrm{~K} \leq T \leq 9.6 \mathrm{~K})$ are shown in Fig. 3. The ambient pressure data are in agreement with previous studies $[1,2]$, and when using the piston cell, the temperature dependences of the magnetic responses under pressure are identical, except for a vertical shift coming from a change in the background that was not subtracted for clarity. Since no changes were detected up to $0.6 \mathrm{GPa}$, a decision was made not to continue to higher pressures in the piston cell, but to shift the experiments to the anvil cell. For the anvil cell, the temperature-dependent background contribution was significantly larger than the signal from the sample. Consequently, the thermal response of the magnetic signal at $T \gtrsim 5 \mathrm{~K}$ is not associated with the sample as it is considered to be outside the dynamic range of detection. Nevertheless, the thermally induced changes at the lower temperatures, $T \lesssim 5 \mathrm{~K}$, are interpreted as arising from the CUEN sample and indicate the peak in the magnetic response appears to be shifted to lower temperatures. These data sets serve as a definitive guide for potential future studies which will need to provide

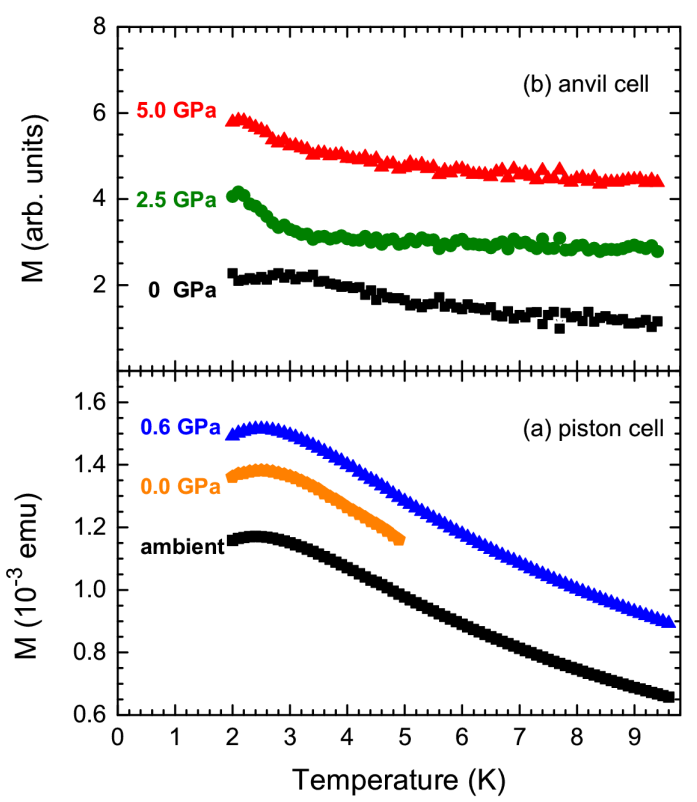

Fig. 3. The temperature dependences of the magnetization, $M$, is shown. In (a), the ambient pressure data were acquired with the sample contained in a holder that was not in the piston pressure cell, and the $0.0 \mathrm{GPa}$ and $0.6 \mathrm{GPa}$ data sets were acquired with the holder inside the pressure cell. In (b), the data obtained with the anvil pressure cell are shown after a significant temperature dependent background was subtracted. 
more isobaric steps to higher pressures $(P \gtrsim 10 \mathrm{GPa})$, to improve signal-to-noise by spanning a wider range of field $\left(\mu_{0} H \gtrsim 5 \mathrm{~T}\right)$, and to extend to lower temperatures $(T \lesssim 400 \mathrm{mK})$.

\section{Summary}

The pressure results using the piston cell, with $P \leq$ $0.6 \mathrm{GPa}$ showed no modifications of the magnetic response for $2 \mathrm{~K} \leq T<9.6 \mathrm{~K}$. Contrastingly, evidence of the peak in the low-field magnetic susceptibility shifting to lower temperatures was detected with the anvil cell for $P=2.5 \mathrm{GPa}$ and $5 \mathrm{GPa}$. These results provide a significant benchmark for establishing the amount of sample that needs to be used when the experiments are conducted at higher pressures.

\section{Acknowledgments}

This work was supported, in part, by the NSF via DMR-1202033 (MWM), DMR-1461019 (UF Physics REU Program), DMR 1157490 (NHMFL), the State of Florida, and CFNT MVEP-Centre of Excellence of the Slovak Academy of Sciences, SAS, and the projects APVV LPP-0202-09 and VEGA 1/0143/13. Instrumentation used with the anvil pressure cell was made possible by the NHMFL User Collaboration Grants Program (JJH), and we gratefully acknowledge Y. Takano, who made the CuTi-alloy available, and S.L. Cooper, who provided details about $\mathrm{SiC}$ anvils.

\section{References}

[1] M. Kajňaková, M. Orendáč, A. Orendáčová, A. Vlček, J. Cernák, O. V. Kravchyna, A. G. Anders, M. Bałanda, J.-H. Park, A. Feher, M. W. Meisel, Phys. Rev. B 71, 014435 (2005).

[2] R. Tarasenko, A. Orendáčová, E. Čižmár, S. Mataš, M. Orendáč, I. Potočňák, K. Siemensmeyer, S. Zvyagin, J. Wosnitza, A. Feher, Phys. Rev. B 87, 174401 (2013).
[3] R. Sýkora, D. Legut, U.D. Wdowik, Acta Phys. Pol. A 126, 50 (2014).

[4] R. Sýkora, D. Legut, J. Appl. Phys. 115, 17B305 (2014).

[5] M.K. Peprah, Ph.D. Thesis, University of Florida, 2015.

[6] A. Eiling, J.S. Schilling, J. Phys. F Met. Phys. 11 623 (1981).

[7] K.V. Kamenev, S. Tancharakorn, N. Robertson, A. Harrison, Rev. Sci. Instrum. 77, 073905 (2006).

[8] K. Kamishima, M. Hagiwara, H. Yoshida, Rev. Sci. Instrum. 72, 1472 (2001).

[9] J. Kamarád, Z. Machátová, Z. Arnold, Rev. Sci. Instrum. 75, 5022 (2004).

[10] Y. Uwatoko, T. Fujiwara, M. Hedo, F. Tomioka I. Umehara, J. Phys. Condens. Matter 17, S1011 (2005).

[11] K. Yokogawa, K. Murata, H. Yoshino, S. Aoyama, Jpn. J. Appl. Phys. 46, 3636 (2007).

[12] M.S. Torikachvili, S.K. Kim, E. Colombier, S.L. Bud'ko, P.C. Canfield, Rev. Sci. Instrum. $\mathbf{8 6}$, 123904 (2015).

[13] G. Giriat, W. Wang, J.P. Attfield, A.D. Huxley, K.V. Kamenev, Rev. Sci. Instrum. 81, 073905 (2010).

[14] D.E. Graf, R.L. Stillwell, K.M. Purcell, S.W. Tozer, High Pressure Res. 31, 533 (2011).

[15] A.D. Chijioke, W. Nellis, A. Soldatov, I.F. Silvera, J. Appl. Phys. 98, 114905 (2005).

[16] S. Buchsbaum, R.L. Mills, D. Schiferl, J. Phys. Chem. 88, 2522 (1984)

[17] J.J. Hamlin, D.E. Jackson, J.J. Hamlin, to be published. 